\title{
Publisher Correction: Single-cell manifold-preserving feature selection for detecting rare cell populations
}

Shaoheng Liang (D), Vakul Mohanty, Jinzhuang Dou, Qi Miao, Yuefan Huang, Muharrem Müftüoğlu, Li Ding, Weiyi Peng (D and Ken Chen (D)

Correction to: Nature Computational Science https://doi.org/10.1038/s43588-021-00070-7, published online 20 May 2021.

In the version of this Article originally published, ref. 64 was missing: 'Liang, S. et al. SCMER: single-cell manifold preserving feature selection. Code Ocean https://doi.org/10.24433/CO.6781338.v1 (2021).' This reference has now been added and is cited at the end of the

Code availability statement instead of the DOI link.

Published online: 1 June 2021

https://doi.org/10.1038/s43588-021-00091-2

(c) The Author(s), under exclusive licence to Springer Nature America, Inc. 2021 